\title{
Haemorrhagic knee- just the tip of an iceberg
}

\author{
Shuaib Ahmed ${ }^{1, *}$, Shishir Murugharaj Suranigi², Nayyar Iqbal ${ }^{3}$, Dharun Raj $^{4}$, Syed Najimudeen ${ }^{5}$ \\ ${ }^{1}$ Senior Resident, ${ }^{\mathbf{2 , 3}, 5}$ Professor \& HOD, ${ }^{4}$ Junior Resident, ${ }^{\mathbf{1 , 2 , 4 , 5}}$ Dept. of Orthopaedics, 3Dept. of General Medicine, Pondicherry \\ Institute of Medical Sciences, Puducherry, India
}

*Corresponding Author:

Email: shuaibahmed99@gmail.com

\begin{abstract}
Fever associated with painful joint and effusion points most of the time towards septic arthritis, probably of bacterial etiology which can also be the first manifestation of infective endocarditis. Septic arthritis is not an uncommon presentation in infective endocarditis. We had an experience with a rare presentation of infective endocarditis where a 15 years old girl came with painful joint effusion. She was evaluated and blood investigations showed elevated ASO titre and total leukocyte count. Transoesophageal echocardiogram revealed infective endocarditis. Having known the rare presentations of infective endocarditis a thorough evaluation of patient should be done in order to not miss the bigger diagnosis such as infective endocarditis.
\end{abstract}

Keywords: Haemorrhagic, Knee, Septic, Arthritis, Infective endocarditis.

\section{Introduction}

Monoarticular knee pain in a paediatric age group directs the clinician towards varied diagnosis. When there is associated fever, this narrows down the differentials to mainly infective pathology.

The reported incidence of patients with infective endocarditis and acute septic arthritis is $15 \% .^{1}$ Septic arthritis may involve one or more joints, and may be the first presenting symptom. ${ }^{2}$

\section{Case Report}

We report a 15 years old girl who presented with pseudoparalysis of right lower limb and painful swelling of the right knee. There was associated fever and tachypnoea for 3 days. Fever was low grade not associated with chills or rigors. There was no history of sore throat or trauma. She gave no history of similar complaints in the past. Clinical examination revealed effusion of right knee joint along with 30 degrees flexion deformity (Fig. 1). There was no lymphadenopathy. Gross systemic examination was normal. Routine blood investigations were done of which showed elevated ASO titre (368 IU) and total leukocyte count $\left(34600 / \mathrm{mm}^{3}\right)$ with neutrophil predominance (85\%). Radiographic assessment of knee joint was normal. Urine examination, blood culture was sent and chest radiograph were also normal. Coagulation profile and screening for connective tissue diseases did not reveal any abnormality. Since there was fever and joint effusion empirical antibiotic (Benzyl Penicillin) was started. After 48 hours of wait there was no growth on culture media. The patient had tachypnoea for which transthoracic echocardiogram was done which was normal and hence infective endocarditis as a probable diagnosis was ruled out. Arthrocentesis revealed minimal blood tinged serous aspirate which showed gram positive cocci on Gram's stain and later Streptococcus pyogenes was grown on culture media. As all these characteristics point towards infective pathology of the knee and a provisional diagnosis of septic arthritis most probably due to streptococcus pyogenes was considered. The patient was then taken up for emergency arthrotomy. Intraoperatively to our surprise there was haemorrhagic effusion in the knee joint and there was no obvious pus (Fig. 2). The effusion and synovium obtained intraoperatively were investigated but did not reveal any organisms. The patient was continued on empirical antibiotic (Benzyl Penicillin). On the seventh day following admission her total count began to show steady decline (dropped to $24000 / \mathrm{mm}^{3}$ ). She needed about four packed cell transfusions as there was drop in the haemoglobin from $9.3 \mathrm{gm} / \mathrm{dl}$ on admission to 6.8 $\mathrm{gm} / \mathrm{dl}$ which was the seventh day post admission. The patient had persistent tachypnoea for which a transoesophageal echocardiogram was done and revealed a small $(3 \times 1 \mathrm{~mm})$ echogenic shadow in the posterior mitral leaflet suggestive of infective endocarditis. Having finally made the diagnosis on the $12^{\text {th }}$ day after admission she was switched to cephalosporin group of antibiotics (ceftriaxone) which showed good clinical response. She was shifted out from the ICU on the $15^{\text {th }}$ day from admission. She was initially started on assisted weight bearing mobilisation with the help of a walker and later mobilised without any walking aids. Hence there was an overall improvement of the patient. 


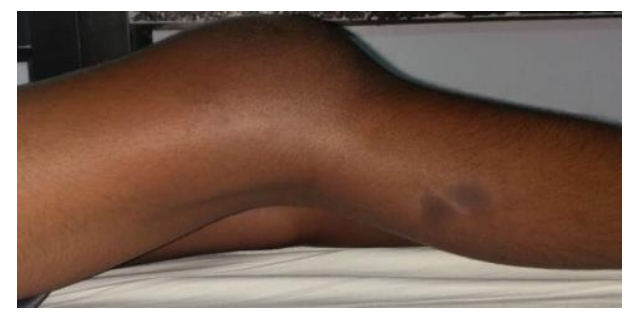

Fig.1: Flexion deformity of the right knee with knee joint effusion

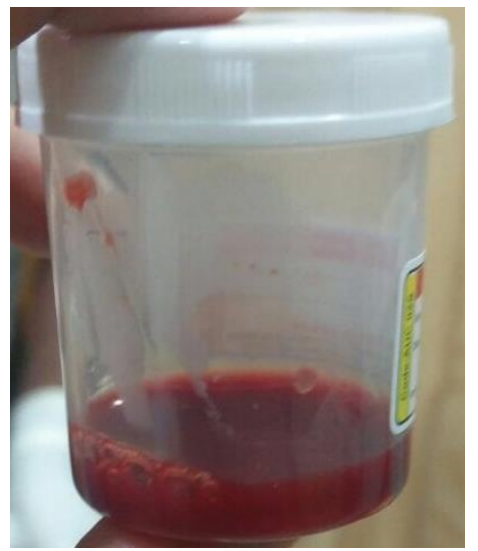

Fig. 2: Haemorrhagic effusion on arthrotomy

\section{Discussion}

Acute septic arthritis is common among paediatric age group. ${ }^{3}$ According to Arnet et $\mathrm{al}^{4}$ in the lower extremity, knee is the most common joint affected preceding hip and ankle.

Symptoms of joint pain, fever and pseudoparalysis clinically points towards septic arthritis. There is always a turbid knee aspirate with frank pus on arthrotomy which was absent in our case.

Haemorrhagic knee effusions were also considered in our differential diagnosis initially. But since this was a female patient and the coagulation profile was normal, blood dyscrasias were ruled out.

Tuberculosis should also be kept in mind knowing that its prevalence in India is quite high. In our case we did not consider it because the course of the disease is quite long with elevated ESR and CRP and classic radiographic features of joint destruction which was absent in our patient.

Musculoskeletal manifestations of infective endocarditis (IE) are difficult to diagnose. Until recently their frequency has been underestimated. Sapico et $\mathrm{al}^{1}$ in their 4-year experience have reported an incidence of 19 to $28 \%$ of patients with IE having musculoskeletal manifestations. The main orthopaedic manifestations were arthralgia, sometimes sequentially affecting several joints, and less often causing arthritis. An acute, sterile synovitis as well as myalgias are also common in bacterial endocarditis.

Bone or joint infections are present in $15 \%$ of cases in some series ${ }^{1}$. Arthralgia and arthritis preferentially involve the major joints and show rapid and complete resolution with antibiotics. The pathogenesis of haemorrhagic joint effusion may be related to the exotoxins released by the infective organism.

Ramaswamy et $\mathrm{al}^{5}$ have found that acute septic arthritis preceded IE in their case report. They confirmed multiple septic arthritis by histopathological examination of synovium, but culture of synovial fluid was sterile.

There are only a few cases of septic arthritis reported during IE mainly due to the predominant cardiac manifestations. Surprisingly in our patient too, the presentation was vice-versa with knee joint haemorrhagic effusion leading to IE involving the mitral leaflet. The sequence of investigation leading to a definitive diagnosis was in a retrograde direction. Baitch et $\mathrm{al}^{6}$ and Omari et $\mathrm{al}^{7}$ have found that the aortic valve and its adjacent ring are more susceptible to abscesses and other cardiac complications are less commonly seen with mitral valve and ring.

\section{Conclusion}

In paediatric patients presenting with knee joint effusion and fever, cardiac screening should always be considered. Such a presentation may be the first clue to IE. Timely diagnosis is very vital as it can avoid major complications causing multiple joints septic arthritis ${ }^{5}$. Early diagnosis will not only help improve the functional outcome but also lessen the hospital stay as in our case.

\section{Financial support: Nil}

This work is an original case report carried out in our hospital by the hereby mentioned authors

\section{Previous presentations: Nil}

\section{References}

1. Sapico FL, Liquete JA, Sarma RJ. Bone and joint infections in patients with infective endocarditis: review of a 4-year experience. Clin Infect Dis 1996;22:783-7.

2. Denis Spelman, Daniel J Sexton; Complications of infective endocarditis cmbi.bjmu.edu.cn.

3. Nicole I, Montgomery, Epps HR. Pediatric Septic Arthritis. Orthop Clin Nor Am Apr 2017;48(2):209-16.

4. Arnett, EN, Roberts, WC. Valve ring abscess in active infective endocarditis: Frequency, location, clues to clinical diagnosis from the study of 95 necropsy patients. Circulation 1976;54:140.

5. Ramaswamy, Sreejith, Sibin et al. Infective endocarditis presenting as multiple septic arthritis- a case report. Research Gate 2013.

6. Baitch A. Recent observations of acute suppurative arthritis. Clin Orthop. 1962;22:157-66.

7. Omari, B, Nelson, RJ, Shapiro, S, et al. Predictive risk factors for periannular extension of native valve endocarditis. Clinical and echocardiographic analyses. Chest 1989;96:1273. 